\title{
Stability Analysis of Milling Process with Variable Spindle Speed and Pitch Angle considering Helix Angle and Process Phase Difference
}

\author{
Gang Jin $\mathbb{D}^{1},{ }^{1}$ Haotian Jiang, ${ }^{1}$ Jianxin Han, ${ }^{1}$ Zhanjie Li, ${ }^{1}$ Hua Li, ${ }^{2}$ and Bing Yan ${ }^{1}$ \\ ${ }^{1}$ Tianjin Key Laboratory of High Speed Cutting and Precision Machining, Tianjin University of Technology and Education, \\ Tianjin 300222, China \\ ${ }^{2}$ Tianjin Jinhang Institute of Technical Physical, Tianjin 300300, China
}

Correspondence should be addressed to Gang Jin; jgang625802@163.com

Received 3 December 2020; Revised 26 January 2021; Accepted 28 January 2021; Published 9 February 2021

Academic Editor: Xiaoliang Jin

Copyright $\odot 2021$ Gang Jin et al. This is an open access article distributed under the Creative Commons Attribution License, which permits unrestricted use, distribution, and reproduction in any medium, provided the original work is properly cited.

Suppression of milling chatter by disrupting regenerative effect is a well-known method to obtain higher cutting stability domain. In this paper, a dynamic model of the milling process with variable spindle speed and pitch angle considering helix angle and process phase difference is presented. Then, an updated semidiscretization method is applied to obtain the stability chart. After the effectiveness of the proposed method is confirmed by comparisons with the previously published works and the time-domain simulations, lots of analyses are conducted to deeply evaluate the influence of the helix angle, the process phase difference, and feed per tooth on milling stability. Results show that the change of helix angle can result in significant stability discrepancies for both high-speed and low-speed regions. Though the process phase difference has the randomness and immeasurability in the practical application, it has an important influence on the stability and will result in a periodic evolution of the stability with a period $\pi$. Also, its recommended values are given for the practical milling process.

\section{Introduction}

Chatter in metal cutting processes is a form of self-excited vibration and always causes lower material removal rates, machining quality, poor accuracy and surface finish, unpleasant noise and sound, accelerated tool wear, and so on in practice. The most powerful source of chatter is regeneration, which is associated with the surface waviness variation during successive cuts. Thus, in order to avoid chatter and achieve high productivity in machining, lots of investigations had been done based on the regeneration mechanism for optimizing the machining conditions in the past few decades.

Generally, the regenerative chatter is modeled and analyzed in the frequency domain or time domain. The most established method for predicting and preventing regeneration chatter is of cutting parameters selection from a stability lobe diagram (SLD), which can predict the stability boundary and distinguish the stable and unstable cutting region. Around this theme, various typical methods, such as zeroth-order approximation method [1], temporal finite element analysis [2], multifrequency solution [3], Chebyshev polynomial approximation [4], semidiscretization method (SDM) [5-7], full-discretization method (FDM) [8, 9], spectral element method [10], have been proposed for more rapidly and accurately predicting the stability chart successively.

Alternatively, some methods focused on enlarging the stable cutting region of the stability chart by disrupting the regenerative effect. One typical example is the utilization of some special cutters, such as variable pitch cutter (VPC) suggested by Slavicek [11]. In this case, the modulation in chip thickness caused by a nonuniform pitch can reduce the vibration under disturbance. Aiming at predicting the stability of milling process with VPC, frequency-domain model $[12,13]$, the Cluster Treatment of Characteristic Roots [14], 
unified SDM [15], improved FDM [16, 17], and improved SDM [18] have been proposed successively. Furthermore, Sims et al. [19], Sims [20], Dombovari and Stepan [21], and Jin et al. $[22,23]$ proposed efficient methods to predict the stability for VPC and variable helix cutter, while Yusoff and Sims [24] optimized the geometry of variable helix tool to suppress regenerative chatter. Besides, the use of serrated cutters [25] also exhibited good capacity and potential for a higher stable depth of cuts.

Another typical example to achieve regenerative chatter disruption is the use of variable spindle speed (VSS), which has a similar mechanism to the use of cutter with variable pitch/helix cutter. Insperger and Stepan [26] and Zatarain et al. [27], respectively, used the SDM and frequency-domain method to construct stability diagrams in the VSS process. Seguy et al. [28] studied VSS in high-speed milling and showed its effective suppression on the unstable domain of the first flip lobe. Long and Balachandran [29] presented a stability treatment for upmilling and downmilling processes with a VSS and discussed the benefits of VSS milling operations. Later, improved SDM [30] and Chebyshev collocation method [31] are presented to efficiently evaluate the process stability in milling with VSS, respectively.

Recently, Jin et al. [32] constructed a new dynamic model to investigate the potential of chatter suppression for the milling process in which the variable pitch cutter and variable spindle speed (VPCVSS) are considered simultaneously. Results show that the combined effect of VPC and VSS on improving the stability region exists and it is more remarkable in certain cases. Obviously, VPCVSS is not a simple combination of VPC and VSS. For modeling, the special characteristics caused by the processes combination, such as time-varying delay, process phase difference, and time-varying feed, significantly increase the difficulty of dynamic modeling, solving, and simulation. For stability impact, the form of the perturbation of the regeneration has realized upgrading to some extent. This upgrade is not a simple superposition of the positive effects of VPC and VSS on system stability but has its own mechanism and phenomenon. However, the research still exists following deficiencies:

(1) The actual feature of a tool, helix angle, is not considered during modeling. However, for a general cutting process, the effect of helix angle is crucially important $[33,34]$. Thus, the related results cannot reflect the influence of the helix angle on the stability, and its real influence is still worthy of exploration and investigation.

(2) The analysis is based on an assumption that the value of the process phase difference is equal to 0 ; however, it is nonzero usually (note that this will be discussed in detail in Section Section 2.1.1). Thus, its influence on system stability needs to be explored further.

(3) The analysis focuses on the influence of the process parameters, that is, the parameters associated with variable tooth and variable speed, on the system stability. However, other important cutting conditions (such as feed rate, feed direction, and helix angle) are not studied yet.
With the above questions in mind, in this paper, a dynamical model of milling process of VPCVSS considering the process phase difference and the helix angle is established for the first time, and then the influence analyses associated with process phase angle, helix angle, and feed per tooth are conducted based on an updated SDM; some relevant engineering suggestions are given. The structure of the paper is as follows: in Section 2, the mathematical model and the method for milling stability are introduced. In Section 3, the method verification and the stability analysis are conducted. In Section 4, conclusions are presented in brief.

\section{Stability Prediction of Milling with VPCVSS}

Generally, the dynamics of the tool-workpiece system in the milling process is described by the models in the form of linear delay-differential equations considering regenerative effect. Based on the number of system modes, these equations usually are single DOF or two DOFs ones. For the former, the workpiece is assumed to be more flexible compared to the rigid tool $[5,35,36]$, but the latter models the tool as a cantilever beam because of the consideration of rigid workpiece [1-3].

Here, for the convenience of the following analysis, the single DOF model introduced in [5] is chosen and its schematic diagram is shown in Figure 1(a), where a helix tool with radius $R$, helix angle $\beta$, and $N$ unequally spaced teeth is rotating at a variable velocity $\Omega(t)$ in rpm, but the workpiece is excited by the milling force at the tooltip. The mathematical representation of this system is given by

$$
m_{x} \ddot{x}(t)+c_{x} \dot{x}(t)+k_{x} x(t)=-F_{x}(t),
$$

where $x(t)$ and $F_{x}(t)$ are the position and cutting force in the $x$ direction. $m_{x}, c_{x}$, and $k_{x}$ are the modal mass, damping coefficient, and spring stiffness, respectively.

2.1. Cutting Force $F_{x}(t)$. Because of the existence of the cutter helix angle, the height of the cutter teeth in the cutting changes all the time. Thus, the cutting force acting on the whole axial cutting height is the integration of differential force with respect to the differential axial depth $d \mathbf{z}$ along the compliant direction of the workpiece, as shown in Figure 1(c). If the nonlinear force model suggested in [32] is considered, the cutting force here can be expressed as

$$
\begin{gathered}
F_{x}(t)=\sum_{j=1}^{N} \int_{0}^{a_{p}} \mathrm{~d} F_{j x}(t, z)=\sum_{j=1}^{N} \int_{0}^{a_{p}} g\left(\phi_{j}\right) \\
\left(\sin \phi_{j} k_{t}+\cos \phi_{j} k_{r}\right) h_{j}^{q}(t) \mathrm{d} z,
\end{gathered}
$$

where $q$ is the nonlinear force coefficient. Influenced by the judgment function $g\left(\phi_{j}\right)$ in equation $(2), F_{x}(t)$ is piecewise continuous [37]. As shown in Figure 1(d), each helix tooth will go through three regions, that is, entry (labeled by (1)), middle of the cut (labeled by (2)), and exit region (labeled by (3)) in a milling process. For one case with the same helix angle and axial depth of cut, there may be two types of such regions due to the different radial depth of cut, as shown in 


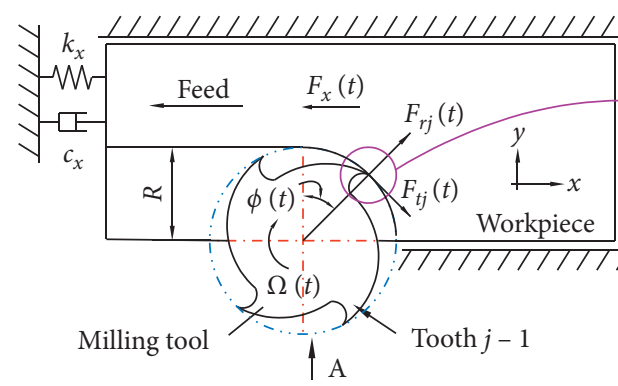

(a)

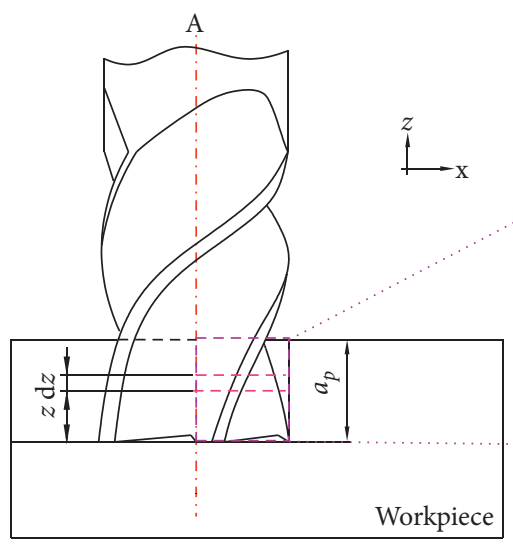

(c)
Waves due to

present vibration

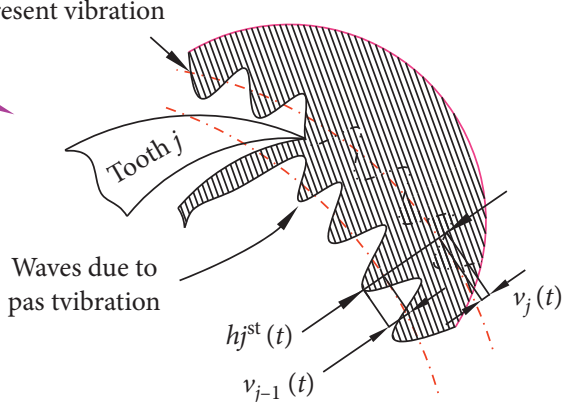

(b)

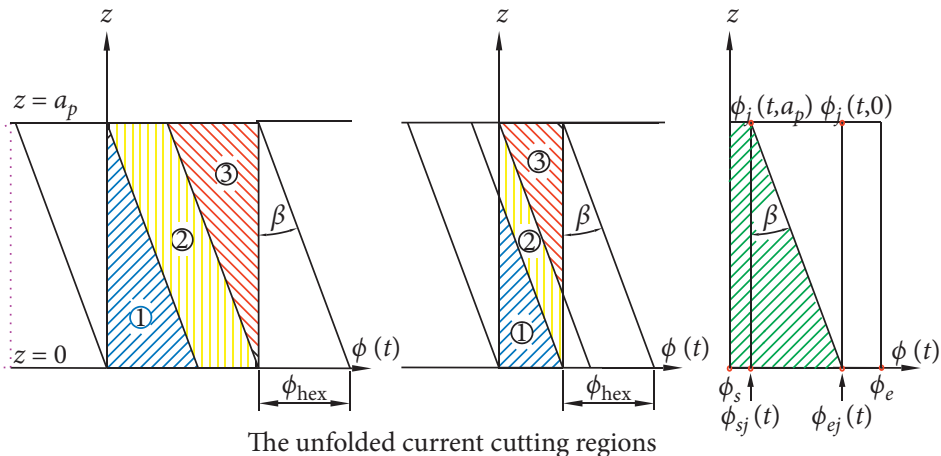

(d)

FIGURE 1: Schematic mechanical model of a single DOF milling system (a), regenerative effect (b), and different regions of cutting caused by the helix angle ((c) and (d)).

the left and middle graphics of Figure 1(d). Obviously, each region corresponds to different calculation methods of the upper and lower limits of integration in equation (2). Here, function $G\left(\phi_{j}\right)$ is introduced instead of the original $g\left(\phi_{j}\right)$, and the unified calculation models for the upper and lower limits of integration; that is, $\phi_{e j}(t)=\min \left(\phi_{j}(t, 0), \phi_{e}\right)$ and $\phi_{s j}(t)=\max \left(\phi_{j}\left(t, a_{p}\right), \phi_{s}\right)$ are utilized (for more details, see $[17,18])$. Then, equation (2) can be transformed into

$$
F_{x}(t)=\gamma \sum_{j=1}^{N} G\left(\phi_{j}\right) \int_{\phi_{s j}(t)}^{\phi_{e j}(t)}\left(\sin \phi_{j} k_{t}+\cos \phi_{j} k_{r}\right) h_{j}^{q}(t) \mathrm{d} \phi
$$

where $\gamma=R / \tan \beta$ and $G\left(\phi_{j}(t)\right)$ is the new judgment function which determines whether the tooth is in contact with the workpiece, defined by

$$
G\left(\phi_{j}(t)\right)= \begin{cases}1, & \text { if } \phi_{s}<\phi_{j}(t)<\phi_{e}+\phi_{\text {hex }} \\ 0, & \text { otherwise }\end{cases}
$$

where $\phi_{s}$ and $\phi_{e}$, respectively, are the exit and start angles which depend on radial immersion ratios $a_{D}$ and milling style (up- or downmilling), $\phi_{\text {hex }}$ is the exit angle difference caused by the helix angle and is equal to $\phi_{\text {hex }}=a_{p} \tan \beta / R$.

2.1.1. Angular Position $\phi_{j}(t)$. Generally, angular position $\phi_{j}(t)$ is the function of helix angle and spindle speed. Here, it is also relevant to the tooth pitch angle. If a linear tooth pitch variation is shown as

$$
\psi=\left[\psi_{1}, \psi_{2}, \ldots, \psi_{N-1}, \psi_{N}\right]=\left[\psi_{0}, \psi_{0}+\Delta \psi, \ldots, \psi_{0}+(N-1) \Delta \psi\right],
$$

where $\psi_{0}=(360 / N)-(N-1) \Delta \psi / 2$, and a sinusoidal spindle speed modulation modeled as

$$
\Omega(t)=\Omega_{0}+\Omega_{1} \sin \left(\frac{2 \pi}{T_{m}} t\right)=\Omega_{0}\left[1+\mathrm{RVA} \cdot \sin \left(\mathrm{RVF} \cdot \frac{2 \pi}{60} \Omega_{0} t\right)\right],
$$

where RVA $=\Omega_{1} / \Omega_{0}$ is the ratio of the speed variation amplitude to the nominal spindle speed and $\mathrm{RVF}=60 /\left(\Omega_{0} T_{m}\right)$ is the ratio of the speed variation frequency to the nominal spindle speed, are considered the same as [32], the angular position $\phi_{j}(t)$ can be defined by

$$
\phi_{j}(t)= \begin{cases}\frac{2 \pi}{60} \int_{0}^{t} \Omega(s) \mathrm{d} s-z \gamma, & \text { if } j=1, \\ \frac{2 \pi}{60} \int_{0}^{t} \Omega(s) \mathrm{d} s+\sum_{i=1}^{j-1} \frac{2 \pi \psi_{i}}{360}-z \gamma, & \text { if } 1<j \leq N .\end{cases}
$$

As a spindle rotates in the machining process, the pitch angle of VPC changes periodically at the rotation period $\tau_{0}=60 / \Omega_{0}$, whereas the spindle speed varies with the modulation period $T_{m}=60 / \Omega_{0} / \mathrm{RVF}$. Therefore, when VSS and VPC are combined, there must be some problems associated with the phase. As shown in Figure 2, one VPC is rotating with $\Omega(t)$, when its 1-th tooth passes through the 


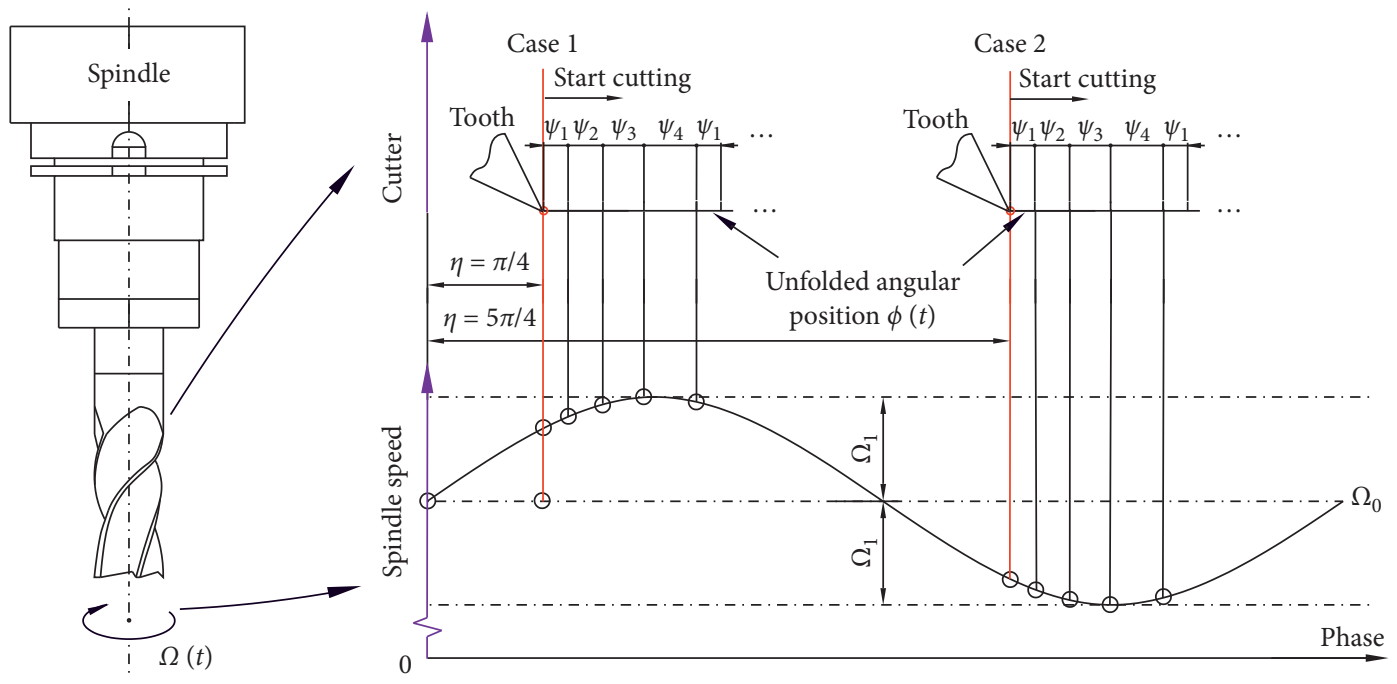

FIGURE 2: Schematic diagram of process phase difference for VPCVSS system.

positive direction of $Y$-axis, that is, the red lines; the value of its corresponding instantaneous spindle speed is not equal to $\Omega_{0}$, but the value is between intervals $\left[\Omega_{0}-\Omega_{1}, \Omega_{0}+\Omega_{1}\right]$, which is depending on the phase of $\Omega(t)$ at this moment. In order to consider this issue, equation (6) is rewritten in a new form including the initial phase as

$$
\Omega(t, \eta)=\Omega_{0}+\Omega_{1} \sin \left(\frac{2 \pi}{T_{m}} t+\eta\right),
$$

where $\eta$ is defined as the process phase difference between VPC and VSS. Obviously, there are kinds of possible $\eta$ in VPCVSS milling process, such as $\pi / 4, \pi$, and $5 \pi / 4$. In other words, the value of $\eta$ is a random one between 0 and $2 \pi$ in the practical application. As a result, the times to pass through any pitch angle $\psi_{j}$ are different when the teeth is rotating at $\Omega(t, \eta)$, for example, Cases (1) and (2) shown in Figure 2. Considering the mechanism of regenerative chatter, it means that $\eta$ will affect the time-varying characteristics of time delays in the milling system. Further, $\eta$ does make sense for milling stability.

2.1.2. Instantaneous Chip Thickness $h_{j}(t)$. For a milling process, the instantaneous chip thickness $h_{j}(t)$ generally consists of two parts, that is, the static chip thickness $h_{j}^{\text {st }}(t)$ mainly determined by the feed per tooth and angular position, and the dynamic displacements $v_{j}(t)$ and $v_{j-1}(t)$, respectively, corresponding to the present tooth $j$ and previous tooth $j-1$ in the radial direction [1]. Thus, $h_{j}(t)$ can be expressed as

$$
\begin{aligned}
h_{j}(t)= & h_{j}^{\mathrm{st}}(t, \eta)+v_{j-1}(t)-v_{j}(t) \\
= & f_{t, j}(t, \eta) \sin \phi_{j}(t)-y\left(t-\tau_{j}(t, \eta)\right) \sin \phi_{j}(t) \\
& +y(t) \sin \phi_{j}(t),
\end{aligned}
$$

where $\tau_{j}(t, \eta)$ represents the time delay between the teeth $j$ and $j-1$, which is mainly affected by the parameters of VPCVSS, such as spindle speed and pitch angles, and $f_{t, j}(t, \eta)$ represents the feed per tooth, which can be described by

$$
f_{t, j}(t, \eta)=v_{f} \tau_{j}(t, \eta)=f_{m} \vartheta_{j}(t, \eta),
$$

where $\vartheta_{j}(t, \eta)=N \psi_{j}\left(1-\mathrm{RVA} \sin \left(\omega_{m} t+\eta\right)\right) / 360$, which is applied to represent the relationship between nominal feed per tooth $f_{m}$ and $f_{t, j}(t, \eta)$.

2.1.3. Time Delays $\tau_{j}(t, \eta)$. Generally, time delay is determined by calculating the rotation time of the tool over the pitch between two successive teeth except for some special cases, such as big cutter runout [15, 37]. For VPCVSS milling, the time delays can be calculated, based on the idea in [5]. Thus, an implicit form including $\tau_{j}(t, \eta)$ is conducted as

$$
\int_{t-\tau_{j}(t, \eta)}^{t} \frac{\Omega_{0}+\Omega_{1} \sin \left(\omega_{m} s+\eta\right)}{60} \mathrm{~d} s=\frac{\psi_{j}}{360} .
$$

Substituting equation (6) into equation (11) and rearranging both sides of equation $(11)$, then, $\tau_{j}(t, \eta)$ can be approximately expressed by the following relationship:

$$
\tau_{j}(t, \eta) \approx \frac{\left(\psi_{j} / \kappa\right)}{6},
$$

where $\kappa=\Omega_{0}+\Omega_{1} \sin \left(\omega_{m} t+\eta\right)$. If $\Omega_{1}$ is small enough compared with $\Omega_{0}$, that is, RVA is small, equation (12) can be expanded and then approximated in the form of

$$
\tau_{j}(t, \eta) \approx \tau_{0, j}-\tau_{1, j} \sin \left(\omega_{m} t+\eta\right),
$$

where $\tau_{0, j}=\psi_{j} / \Omega_{0} / 6$ and $\tau_{1, j}=\tau_{0, j}$ RVA. Here, it should be noted that the approximation errors of equation (13) increase with the increase of RVA. For small RVA, for example, $\mathrm{RVA}=0.2$, there are also errors although the effects of errors on the stability lob may be ignored.

Substituting equations (7) and (9) into equation (3) and considering equation (1), then linearizing the consequent 
equation on the basis of the ideal in [5], a linear time- with periodic DDE can be given by

$$
m_{x} \ddot{x}(t)+c_{x} \dot{x}(t)+k_{x} x(t)=-\varepsilon \sum_{j=1}^{N} K_{j}(t)\left[x(t)-x\left(t-\tau_{j}(t, \eta)\right)\right],
$$

$$
\begin{aligned}
\varepsilon & =\gamma q, \\
K_{j}(t) & =f_{t, j}^{q-1}(t, \eta) G\left(\phi_{j}\right) \int_{\phi_{s j}(t)}^{\phi_{e j}(t)}\left[k_{t} \sin ^{q} \phi_{j}(t) \cos \phi_{j}(t)+k_{r} \sin ^{q+1} \phi_{j}(t)\right] \mathrm{d} \phi,
\end{aligned}
$$

2.2. Updated SDM. SDM is a common method often applied to the stability analysis of various DDEs where the time delayed terms are approximated by a piecewise constant function while the current time terms are left unchanged. Here, the method is presented for the case of VPCVSS.

However, to ensure that equation (14) is periodic and applicable to SDM, the ratio of the spindle modulation period $T_{m}$ and the rotation period $\tau_{0}$ in the current system must be a rational number, that is, $q_{1} T_{m}=q_{2} \tau_{0}$ where $q_{1}$ and $q_{2}$ are relative prime numbers [5]. Thus, the system period $T_{c}$ can be expressed as

$$
\begin{array}{r}
T_{c}=q_{1} T_{m} \\
\quad \text { or } q_{2} \tau_{0} .
\end{array}
$$

By introducing the system vector $\mathbf{U}(t)=(x(t), \dot{x}(t))^{T}$ and using Cauchy transformation, the second-order DDE, that is, equation (14) can be written as a system of the firstorder DDE:

$$
\begin{aligned}
& \dot{U}(t)=\mathbf{A}(t) \mathbf{U}(t)+\sum_{j=1}^{N} \mathbf{B}_{j}(t) \mathbf{U}\left(t-\tau_{j}(t, \eta)\right), \\
& \mathbf{A}(t)=\mathbf{A}\left(t+T_{c}\right), \\
& \mathbf{B}_{j}(t)=\mathbf{B}_{j}\left(t+T_{c}\right),
\end{aligned}
$$

with

$$
\begin{aligned}
\mathbf{A}(t) & =\left[\begin{array}{cc}
0 & 1 \\
-\omega_{n x}^{2}-\frac{\varepsilon l(t)}{m_{x}} & -2 \zeta_{x} \omega_{n x}
\end{array}\right], \\
\mathbf{B}_{j}(t) & =\left[\begin{array}{cc}
0 & 0 \\
\varepsilon \sum_{j=1}^{N} \frac{k_{j}(t)}{m_{x}} & 0
\end{array}\right], \\
l(t) & =\sum_{j=1}^{N} k_{j}(t),
\end{aligned}
$$

where $\omega_{n x}=\sqrt{k_{x} / m_{x}}$ and $\zeta_{x}=c_{x} /\left(2 \omega_{n x} m_{x}\right)$ are the angular natural frequency and the damping ratio.
The first step of SDM is the construction of the discrete time interval $\left[t_{i}, t_{i+1}\right]$ by dividing its period $T_{c}$ into $k$ number with length $\Delta t$, that is, $\Delta t=T_{c} / k$. Then, the time-varying delay $\tau_{j}(t, \eta)$ in equation (17) for the interval $\left[t_{i}, t_{i+1}\right]$ can be written in an average form based on the ideal in [5] as

$$
\tau_{i, j}=\frac{1}{\Delta t} \int_{t_{i}}^{t_{i+1}} \tau_{j}(t, \eta) \mathrm{d} t=\tau_{0, j}-\tau_{1, j} c_{i}
$$

where

$$
c_{i}=\frac{k \nu}{2 \pi} \int_{i 2 \pi / k / v+\eta}^{(i+1) 2 \pi / k / v+\eta} \sin (t) \mathrm{d} t, \quad i=0,1, \ldots, k-1\left(\nu=\frac{T_{m}}{T_{c}}\right) .
$$

Then, substituting equation (19) into equation (17), equation (17) can be approximated in $\left[t_{i}, t_{i+1}\right]$ as new form yields as

$$
\dot{U}(t)=\mathbf{A}_{i} \mathbf{U}(t)+\sum_{j=1}^{N} \mathbf{B}_{i, j}\left(\alpha_{i, j} \mathbf{U}_{i-m_{i, j}+1}+\chi_{i, j} \mathbf{U}_{i-m_{i, j}}\right)=\mathbf{A}_{i} \mathbf{U}(t)+\mathbf{W}_{i},
$$

with

$$
\begin{aligned}
\mathbf{A}_{i} & =\frac{1}{\Delta t} \int_{t_{i}}^{t_{i+1}} \mathbf{A}(t) \mathrm{d} t, \\
\mathbf{B}_{i, j} & =\frac{1}{\Delta t} \int_{t_{i}}^{t_{i+1}} \mathbf{B}_{j}(t) \mathrm{d} t, \\
\alpha_{i, j} & =\frac{\left(m_{i, j} \Delta t+\Delta t / 2-\tau_{i, j}\right)}{\Delta t}, \\
\chi_{i, j} & =1-\alpha_{i, j}, \\
\mathbf{U}_{i-m_{i, j}+1} & =\mathbf{U}\left(t_{i-m_{i, j}+1}\right), \\
\mathbf{U}_{i-m_{i, j}} & =\mathbf{U}\left(t_{i-m_{i, j}}\right), \\
m_{i, j} & =\operatorname{int}\left(\tau_{i, j} / \Delta t+1 / 2\right),
\end{aligned}
$$

where int $\left({ }^{*}\right)$ indicates the operation that rounds positive number towards zero. For the initial value $\boldsymbol{U}\left(t_{i}\right)=\boldsymbol{U}_{i}$, the solution of equation (21) is known: 


$$
\mathbf{U}(t)=e^{\mathbf{A}_{i}\left(t-t_{i}\right)}\left[\mathbf{U}_{i}+\mathbf{A}_{i}^{-1} \mathbf{W}_{i}\right]-\mathbf{A}_{i}^{-1} \mathbf{W}_{i} .
$$

Substituting $t=t_{i+1}$ and $\mathbf{U}\left(t_{i+1}\right)=\mathbf{U}_{i+1}$ into the solution leads to

$$
\mathbf{U}_{i+1}=\mathbf{Q}_{i} \mathbf{U}_{i}+\sum_{j=1}^{N} \mathbf{M}_{i, j, \alpha} \mathbf{U}_{i-m_{i, j}+1}+\mathbf{M}_{i, j, \chi} \mathbf{U}_{i-m_{i, j}}
$$

where $\quad \mathbf{Q}_{i}=e^{\mathbf{A}_{i} \Delta t}, \quad \mathbf{M}_{i, j, \alpha}=\left(e^{\mathbf{A}_{i} \Delta t}-\mathbf{I}\right) A_{i}^{-1} \mathbf{B}_{i, j} \alpha_{i, j}, \mathbf{M}_{i, j, \chi}=$ $\left(e^{\mathbf{A}_{i} \Delta t}-\mathbf{I}\right) \mathbf{A}_{i}^{-1} \mathbf{B}_{i, j} \chi_{i, j}$.

Defining the maximum value of $m_{i, j}$ by $n=\max \left(m_{i, j}\right)$ and state augmentation of equation (24) results in the $2(n+1)$ dimensional discrete map:

$$
\mathbf{V}_{i+1}=\mathbf{Z}_{i} \mathbf{V}_{i}
$$

with

$$
\begin{aligned}
\mathbf{V}_{i} & =\operatorname{col}\left(\mathbf{U}_{i}, \mathbf{U}_{i-1}, \ldots, \mathbf{U}_{i-n+1}, \mathbf{U}_{i-n}\right) \\
\mathbf{Z}_{i} & =\left[\begin{array}{cccccc}
Q_{i} & 0 & \cdots & 0 & 0 & 0 \\
\mathbf{I} & 0 & \cdots & 0 & 0 & 0 \\
0 & \mathbf{I} & \cdots & 0 & 0 & 0 \\
\vdots & \vdots & \ddots & \vdots & \vdots & \vdots \\
0 & 0 & \cdots & \mathbf{I} & 0 & 0 \\
0 & 0 & \cdots & 0 & \mathbf{I} & 0
\end{array}\right]+\sum_{j=1}^{N}\left[\begin{array}{cccccc}
0 & \cdots & \mathbf{M}_{i, j, \alpha} & \mathbf{M}_{i, j, \chi} & \cdots & 0 \\
0 & \cdots & 0 & 0 & \cdots & 0 \\
0 & \cdots & 0 & 0 & \cdots & 0 \\
\vdots & \vdots & \ddots & \vdots & \vdots & \vdots \\
0 & \cdots & 0 & 0 & \cdots & 0 \\
0 & \cdots & 0 & 0 & \cdots & 0
\end{array}\right] .
\end{aligned}
$$

The horizontal position of the discrete input matrices $M_{i, j, \alpha}$ and $M_{i, j, \chi}$ in the right side of equation (27) begins from the column of $2 m_{i, j}-1$ and $2 m_{i, j}+1$, respectively. Here, it should be noted that if one reduction about the matrix $\mathbf{V}_{i}$ is carried out by removing the delayed values of the velocities, like [7], the size of vector $\mathbf{V}_{i}$ can be decreased to $n+2$, the horizontal position of $M_{i, j, \alpha}$ and $M_{i, j, \chi}$ will change from the column of $m_{i, j}+1$ and $m_{i, j}+2$, respectively.

The Floquet transition matrix over the principal period $T_{c}$ is approximated by coupling the solutions of $k$ successive time intervals $\Delta t$ :

$$
\Phi=\mathbf{Z}_{k-1} \mathbf{Z}_{k-2}, \ldots, \mathbf{Z}_{1} \mathbf{Z}_{0} .
$$

Stability of the investigated system is determined by the eigenvalues of the transition matrix $\Phi$. The system is stable if all eigenvalues of $\Phi$ are in modulus less than 1 .

The main advantage associated with the method in this paper can be summarized as follows:

(1) Compared with the original algorithm $[5,6,36]$, the method in this paper can be applied not only to variable pitch milling cutter or variable speed milling process but also to their composite systems. Mathematically, it is suitable for the stability solution of DDEs with multiple variable delays.

(2) Due to the addition of equations (16) and (20), the method in this paper is also applicable to some special cases, where number 1 cannot be divisible by the values of RVF, such as $R V F=0.22,0.3$, or 0.4 . For the above cases, this method is applicable, but the method in [32] is not.

In addition, it should be noted that the 1-DOF VPCVSS model is proposed in this paper; however, the milling system belongs to the 1-DOF model in general. Thus, to realize a conversion from 1-DOF one to 2-DOF one [38], some modifications need to be carried out as follows: (a) the system equation (equation (1)) should consider the dynamics in both $X$ and $Y$ directions instead of the original $X$. (b) Correspondingly, the cutting force model in $X$ and $Y$ directions (equations (2) and (3)) should be constructed. (c) The dynamic cutting force thickness (equation (9)) is changed to a coupled model considering $X$ and $Y$ directions. (d) The system equation (equation (17)) and the corresponding state matrix are changed from two dimensions to four ones. (e) Equations (25) and (26) change from 2(n+1) to $4(n+1)$ or $2 n+4$ if the delayed values of the velocities are removed, thus, the horizontal position of the discrete input matrices $M_{i, j, \alpha}$ and $M_{i, j, \chi}$ in equation (27), respectively, begins from the column of $4 m_{j, k, i}-3$ and $4 m_{j, k, i}+1$ or $2 m_{j, k, i}+1$ and $2 m_{j, k, i}+3$.

\section{Stability Analysis and Discuss}

In this section, the verification of the proposed method is carried out firstly. Then, the influence of the cutting conditions, that is, the process phase angle, the helix angle, and the feed per tooth on the stability of the VPCVSS process is investigated in detail. Note that besides the influence parameters shown in respective figure names, other parameters in the following calculation example are as follows: upmilling, the cutting-force exponent is $q=0.75$, the cutting-force coefficients are $k_{t}=107 \times 10^{6} \mathrm{~N} / \mathrm{m}^{1+q}$ and $k_{r}=40 \times 10^{6} \mathrm{~N} / \mathrm{m}^{1+\mathrm{q}}$, the mode mass is $m_{x}=3.1663 \mathrm{~kg}$, the natural frequency is $\omega_{n}=400 \mathrm{~Hz}$, damping ratios are $\zeta_{x}=0.02$, the number of the cutter teeth is $N=4$, the tool radius is $R=9.525 \times 10^{-3} \mathrm{~m}$, and pitch angles are $\left.\psi=[75,85,95,105]^{\circ}\right)$. Here, it must be emphasized and noted that the VPC used in this paper belongs to an asymmetric cutter. Correspondingly, its dynamic balancing ability is poor and this will be more significant in high-speed cutting. However, this problem cannot be considered in the 
following calculation examples, so there may be some prediction errors associated with cutting stability in the high-speed region.

3.1. Model Verification. In this section, the validity of the proposed method will be carried out by comparing with previous works and the time-domain simulations.

To illustrate the performance of the proposed approach, the related works on VSS in [5] and on VPCVSS in [32] are considered first. Based on the main calculation parameters in $[5,32]$, the proposed method is utilized to calculate the SLDs for both VSS and VPCVSS cases with two different helix angles under two different radial depths of cut. The related results are shown in Figures 3(a)-3(h), where (a)-(d) and (e)-(h), respectively, correspond to VSS and VPCVSS, whilst the stability boundary labeled by blue and red dotted lines are, respectively, associated with the cases of helix angle $\beta=$ $0^{\circ}$ and $30^{\circ}$

If comparing the SLDs marked by blue lines in Figures 3(a) and 3(b) with those in Figures 5.17(a) and (b) in [5], and comparing the SLDs marked by the red dotted lines in Figures 3(e)-3(g) with those in Figures 4(a) and 4(b) and 11 (b) in [32], one can see that their calculation results are in good agreement obviously. This indicates that the proposed method has good reliability and accuracy in predicting the stability for both VSS and VPCVSS processes when the helix angle is $\beta=0^{\circ}$.

Comparing all the blue stability boundaries with the red ones in Figures 3(a)-3(h), it can be found that, no matter for VSS (Figures $3(\mathrm{a})-3(\mathrm{~d})$ ) or VPCVSS (Figures $3(\mathrm{e})-3(\mathrm{~h})$ ) milling processes, the change of helix angle can result in significant stability difference.

In order to effectively verify the above predicting trend of stability caused by helix angle, one updated time-domain program (or called time marching solution) is utilized. It should be noted that this program is mainly crafted on the basis of [39], and its application range is expanded from the stability prediction for a single case of VPC to that of VPCVSS. The most critical operation is that the rotation angle is discrete uniformly for one system cycle but time period. Based on the program, detailed information about the amplitude of the vibrations can be obtained and the contour plot of peak-to-peak (PTP) [39] vibration can be generated for a range of spindle speed and axial depth of cut combinations. Therefore, the appearance of nonlinear vibration (chatter) can be well grasped by examining the abrupt change of vibration displacement with the axial depth of cut increasing uniformly. Remarkably, this program can take into account teeth jumping out of contact, workpiece dynamics, regeneration effect, pitch angles, helix angles, and spindle speed variations simultaneously.

Based on the above program, time-domain simulations for the plot of PTP are carried out under conditions the same as those of the red stability boundaries in Figures 3(g) and $3(\mathrm{~h})$, and the related results are shown in Figures 3(i) and 3(j). Obviously, the prediction results based on the proposed method are in accordance with those by the time-domain simulations. This proves the validity and reliability of the proposed method when the helix angles are considered. On the other hand, there are also some little differences between their results. The reason is that the above time-domain simulations can consider the effect of loss-of-contact character on milling dynamics but the proposed method cannot.

In order to detect the stability difference caused by the helix angle more intuitively, two special cutting points are selected, that is, point " $A$ " in Figure $3(\mathrm{~g})\left(\Omega_{0}=1600 \mathrm{rpm}\right.$, $\left.a_{p}=48 \mathrm{~mm}\right)$ and point " $\mathrm{B}$ " in Figure $3(\mathrm{~h})\left(\Omega_{0}=13000 \mathrm{rpm}\right.$, $\left.a_{p}=27 \mathrm{~mm}\right)$. One can see from the associated SLDs that points " $A$ " and " $B$ " are unstable in the cases of $\beta=0$; however, they will turn to the stable ones when the helix angle changes to $\beta=30^{\circ}$.

Their vibration information is simulated in the time domain and the results associated with the cutting vibration time history and periodic points, Poincare section, and nonuniform fast Fourier transform (NuFFT) are shown in Figure 5. Note that Figures 5(a) and 5(c), respectively, correspond to points " $\mathrm{A}$ " and " $\mathrm{B}$ " under $\beta=0^{\circ}$, but Figures $5(\mathrm{~b})$ and $5(\mathrm{~d})$ correspond to $\beta=30^{\circ}$. Consequently, the associated stability states are as follows:

(i) Figures 5(a) and 5(c) exhibit two unstable processes due to the fact that the period-doubling (characterized by two points in the Poincare section shown as the middle graphic in Figure 5(a)) and quasiperiodic (characterized by the appearance of a circle map for Poincare section shown as the middle graphic in Figure 5(c)) bifurcation occur, respectively. Meanwhile, the large amplitude vibrations occur at chatter frequency (CF).

(ii) Figures 5(b) and 5(d) exhibit two stable processes because they are both characterized by the single fixed point for Poincare mapping (see the middle graphics in Figures 5(b) and 5(d)). There are only modulation frequency (MF) and its harmonics in NuFFTs, and the clearly smaller vibration peaks compared with their counterparts.

Obviously, not to mention CFs, there are so many other frequencies in Figure 5, especially for Figures 5(a) and 5(b). The reason is that the modulated frequency is equal to $\Omega_{0} \mathrm{RVF} /\left(60 q_{1}\right)$ and its harmonics equal to $n \Omega_{0} \mathrm{RVF} /\left(60 q_{1}\right)$, with $n=1,2, \ldots$ appearing now. As a result, their values are about 5.33n for Figures 5(a) and 5(b) and $108.33 n$ for Figures 5(c) and 5(d), respectively.

All in all, the results based on the theoretical calculation and the simulations are consistent.

3.2. Influence of the Process Phase Difference. Because the VPCVSS system is a combination of VPC and VSS, there must be the process phase differences between VPC and VSS as shown in Figure 2. In fact, the value of $\eta$ is almost impossible to be zero in practice; therefore, whether the conclusion based on the assumption of $\eta=0$ in [32] is universal or not needs further discussion and research.

Starting with above problem, to further evaluate the effect of $\eta$ on cutting stability, the SLDs corresponding to 


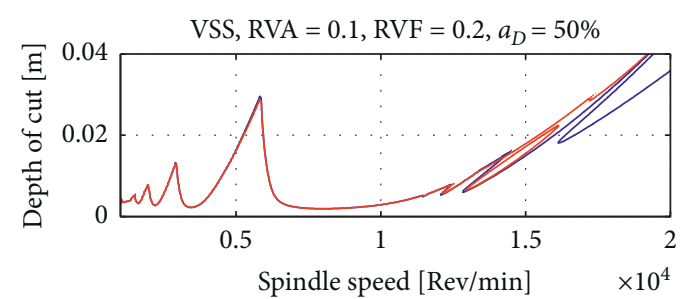

$\begin{aligned}-\beta & =0^{\circ} \\ -\beta & =30^{\circ}\end{aligned}$

(a)

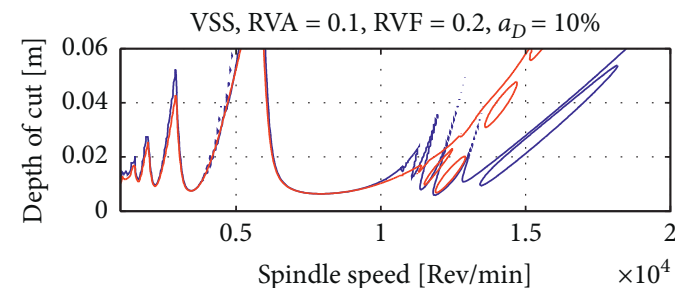

$-\beta=0^{\circ}$

... $\beta=30^{\circ}$

(c)

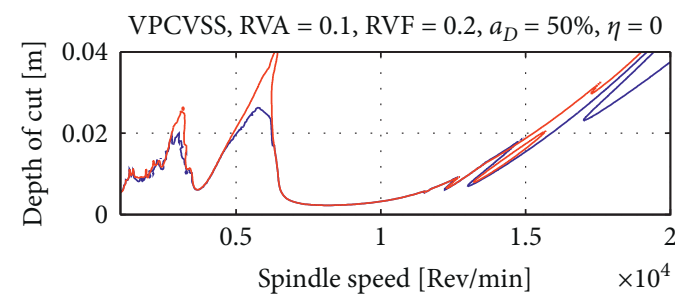

$-\beta=0^{\circ}$

... $\beta=30^{\circ}$

(e)

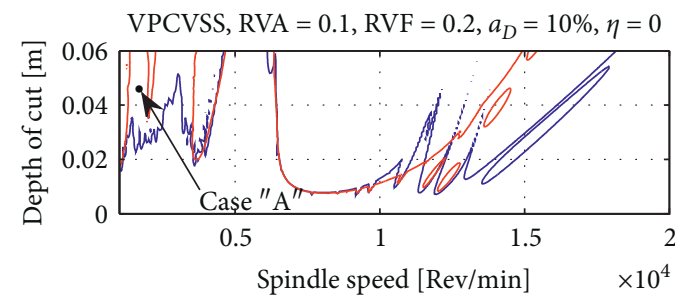

$-\beta=0^{\circ}$

... $\beta=30^{\circ}$

(g)

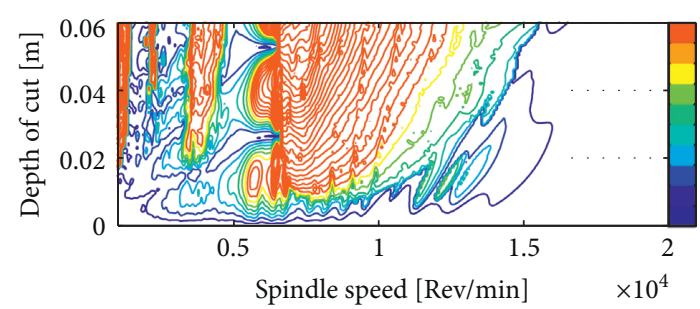

(i)

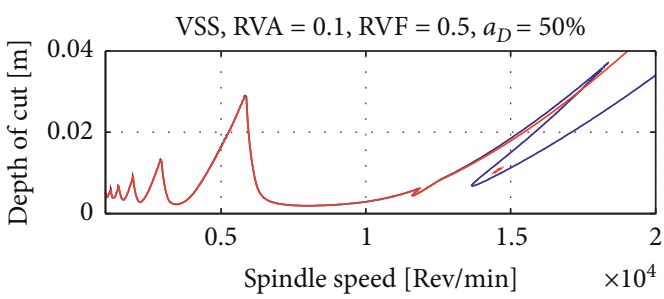

$\begin{aligned}-\beta & =0^{\circ} \\ -\beta & =30^{\circ}\end{aligned}$

(b)

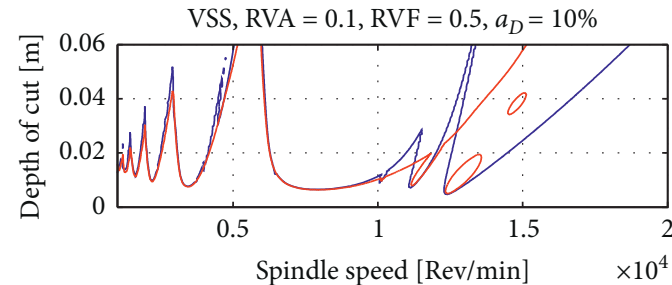

$-\beta=0^{\circ}$

... $\beta=30^{\circ}$

(d)

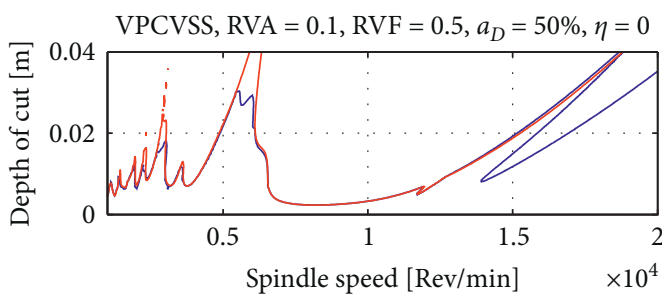

$-\beta=0^{\circ}$

..- $\beta=30^{\circ}$

(f)

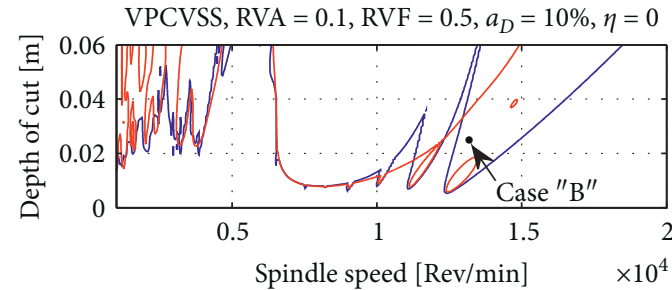

$-\beta=0^{\circ}$

.. $\beta=30^{\circ}$

(h)

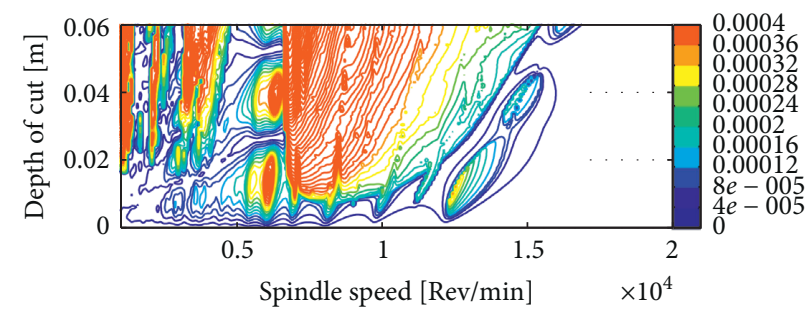

(j)

FIGURE 3: Stability diagram of VSS and VPCVSS systems under different cutting parameters. (a)-(h): obtained by the analytic method; (i)-(j): obtained by time-domain simulation. 


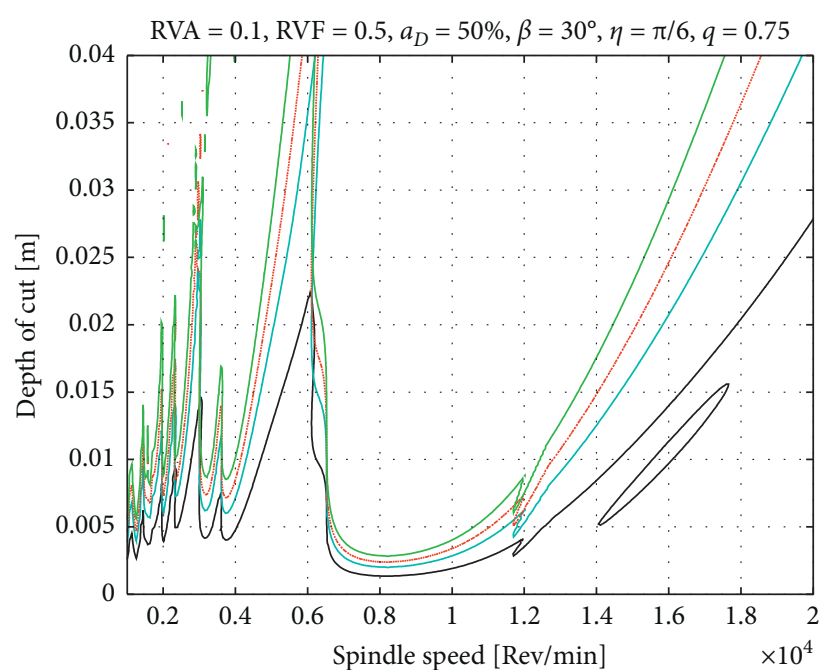

$f_{m}=0.01 \mathrm{~mm} /$ tooth $\quad \cdots f_{m}=0.10 \mathrm{~mm} /$ tooth
$-f_{m}=0.05 \mathrm{~mm} /$ tooth $\quad-f_{m}=0.20 \mathrm{~mm} /$ tooth

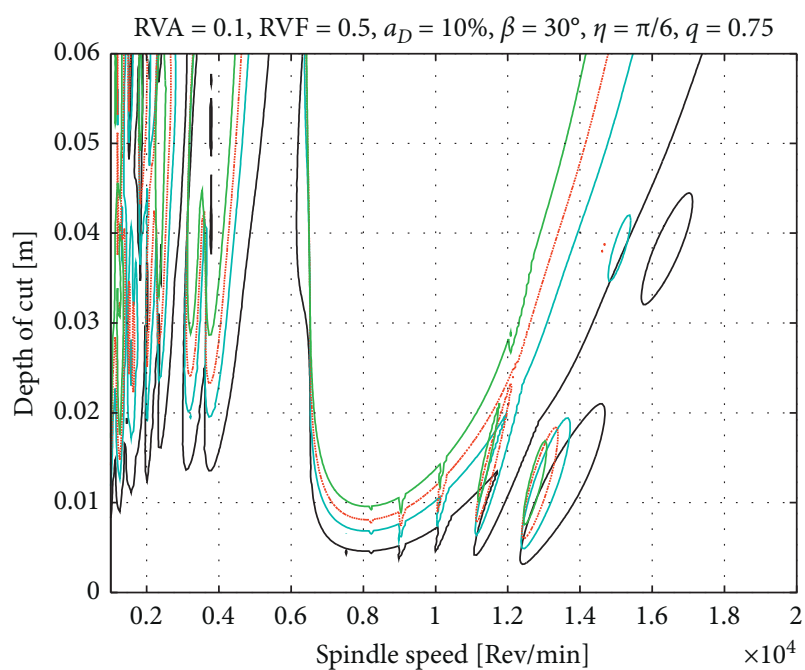

- $f_{m}=0.01 \mathrm{~mm} /$ tooth $\quad \cdots . . . f_{m}=0.10 \mathrm{~mm} /$ tooth

(a)

(b)

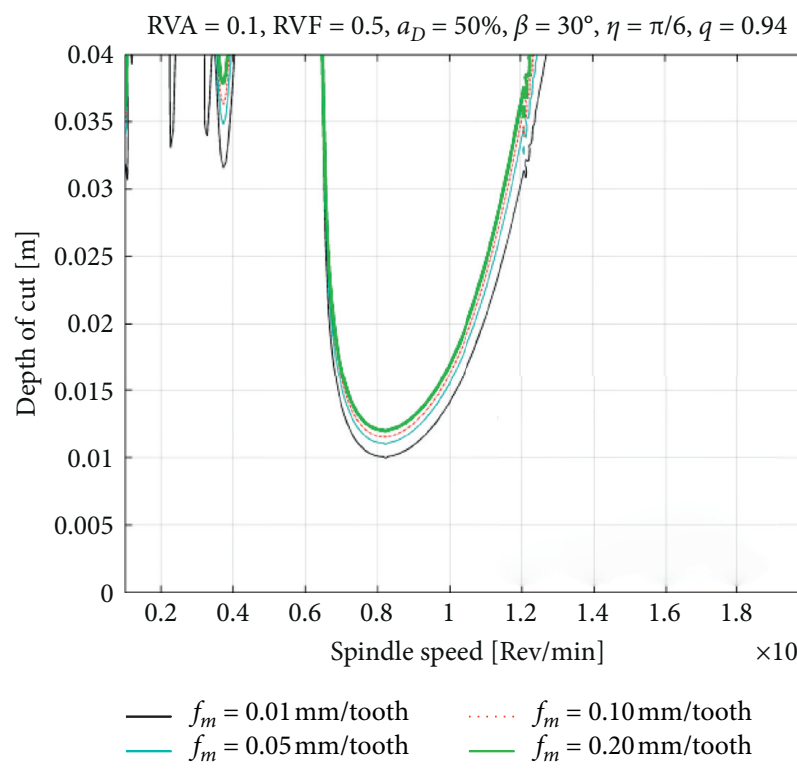

(c)

Figure 4: Effect of feed per tooth on milling stability under different situations. (a) $a_{D}=50 \%, q=0.75$; (b) $a_{D}=10 \%, q=0.75$; (c) $a_{D}=50 \%$, $q=0.94$.

four special process phase differences (i.e., $\eta=0, \pi / 2, \pi$, and $3 \pi / 2$ ) under the large and small radial depth of cut, are calculated and shown in Figures 6(a) and 6(b). It can be seen that there are only two stability curves with obvious difference for every SLD: one is the overlapping stability curves corresponding to $\eta=0$ and $\pi$ (see the blue line and red dotted line) and the other corresponding to $\eta=\pi / 2$ and $3 \pi / 2$ (see the blue line and green dotted line). From a more detailed observation, it can be found that when $\eta$ changes from 0 to $\pi / 2$, the system stability is evolving gradually. At $\eta=\pi / 2$, the evolution difference reaches the peak. Then, $\eta$ is increasing from $\pi / 2$ to $\pi$, its stability continues to evolve gradually; however, the difference decreases on the whole.
Finally, when $\eta=\pi$, its stability is the same as the case of $\eta=0$. Obviously, there are two kinds of limit shapes of the SLDs, that is, ones at $\eta=0$ or $\pi$ and the others at $\eta=\pi / 2$ or $3 \pi / 2$.

In order to evaluate the impact of $\eta$ in more detail, four different $\eta$ are chosen in the interval $[0, \pi / 2]$ corresponding to Figure $6(\mathrm{a})$ and the interval $[\pi / 2, \pi]$ corresponding to Figure 6(b), respectively, and the related SLDs are calculated and shown in Figures 6(c) and 6(d). It can be seen that, for the above intervals, $\eta$ always plays an important role and results in a gradual change of SLDs from one limit to another. If this trend is extended to the whole value domain of $\eta$, one can easily know that the limit shapes of the SLDs 

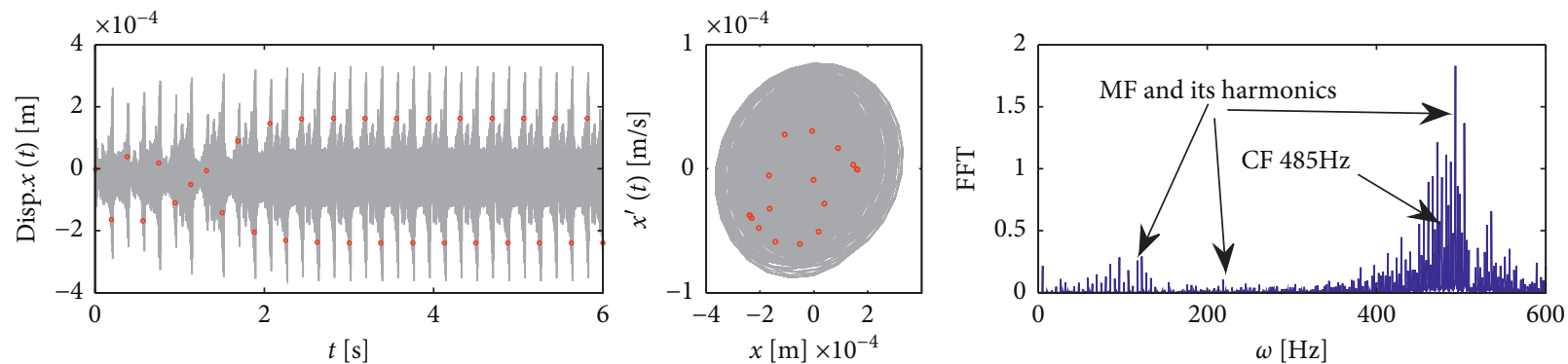

(a)
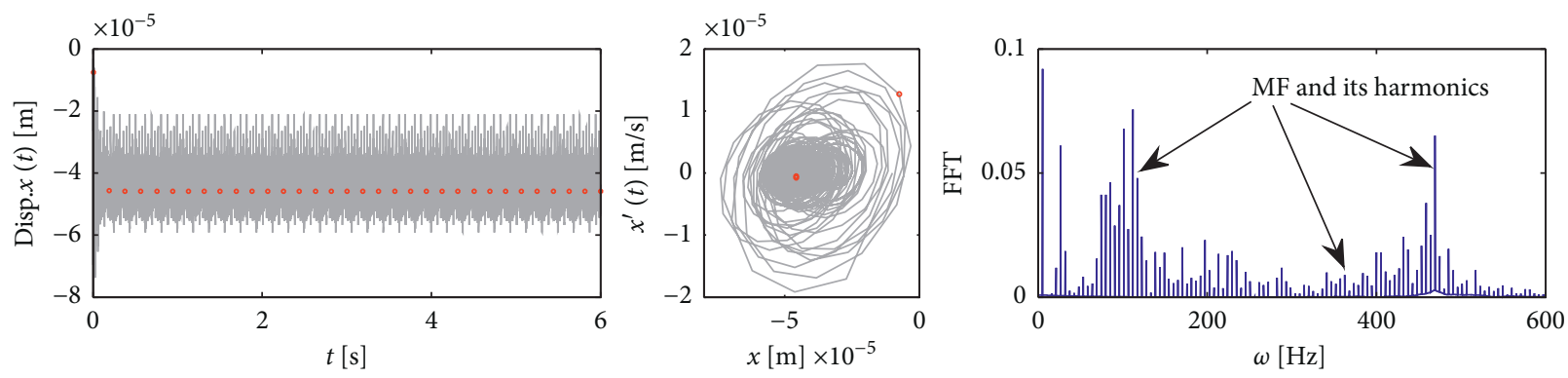

(b)
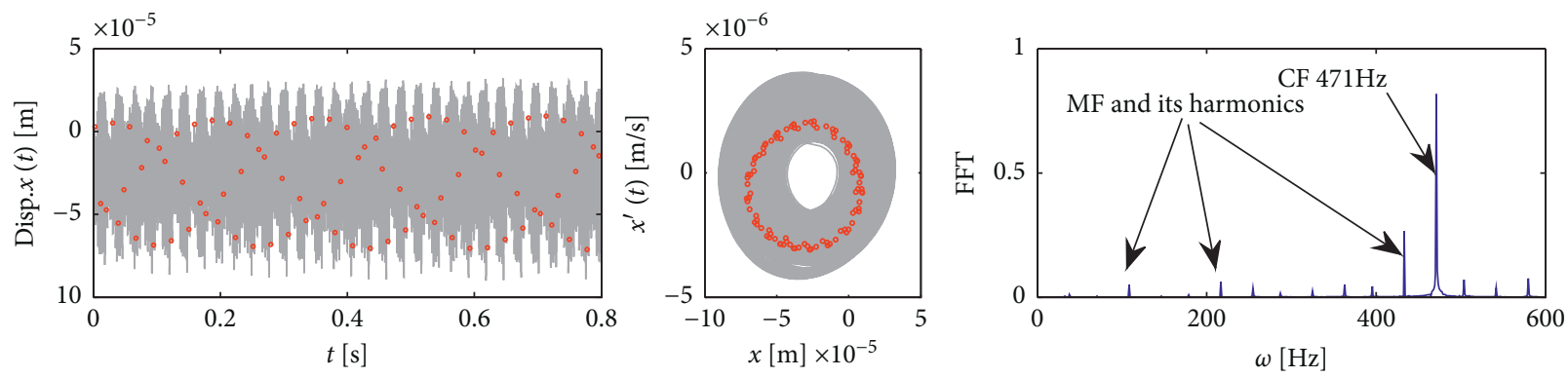

(c)
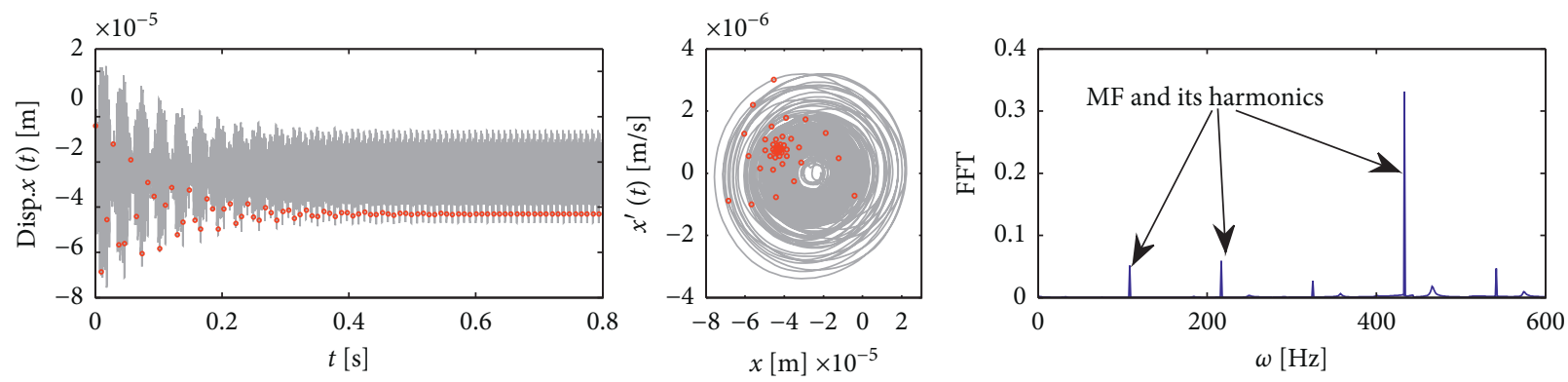

(d)

FIgURE 5: The information associated with the continuous and $1 / T_{c}$-sampled time histories of vibration displacement ( $x$ ), the Poincare section, and the Fast Fourier Transform for points "A" and "B" in Figures 3(g) and 3(h) under different helix angles $\beta$. (a, c) $\beta=0^{\circ}=0^{\circ}$; (b, d) $\beta=90^{\circ}$.

under the influence of $\eta$, respectively, occur at $\eta_{a}=n \pi-\pi$ and $\eta_{b}=n \pi-\pi / 2(n=1,2, \ldots)$, and $\eta$ obviously leads to the periodic evolution of system stability whose period is $\pi$. This is attributed to the following reason. For the VPCVSS processes described in this article, their time delays exist in the form of sine waves as shown in Figures 6(c) and 6(d) (note that they are corresponding to the SLDs in Figures 6(e) and $6(\mathrm{f})$, resp.). When the values of $\eta$ are varying, the waves will move along the time axis (shown as the red lines). As a result, the combinations of the multiple time delays in the milling system will be altered, the stability nature will also be changed consequently.

In view of the significant influence of $\eta$ on cutting stability, it is necessary to consider it for a VPCVSS process. However, as shown in Figure 2, it is obvious that the value of $\eta$ is random and unmeasured in the actual machining process. Considering its periodic influence on the cutting stability region and the variation limit of the SLDs, one can 


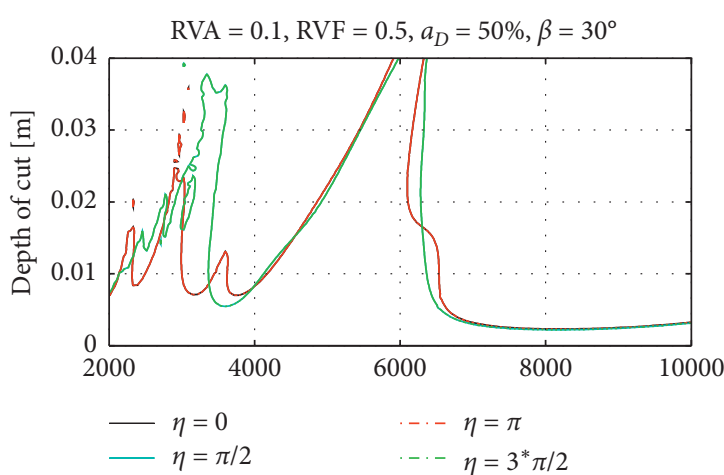

(a)

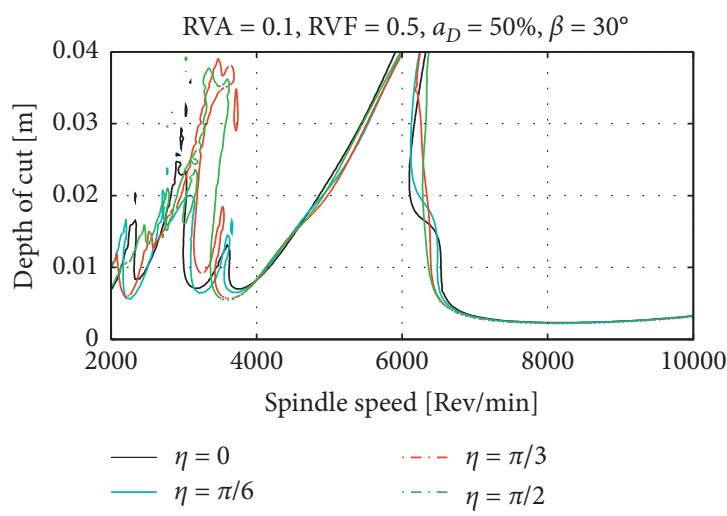

(c)

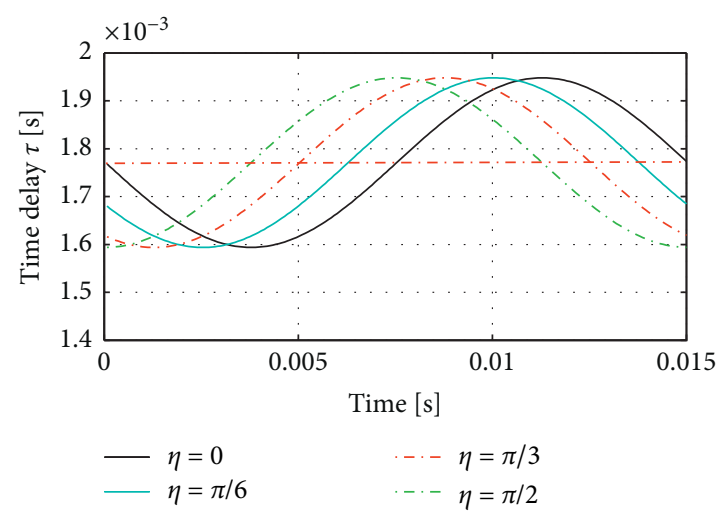

(e)

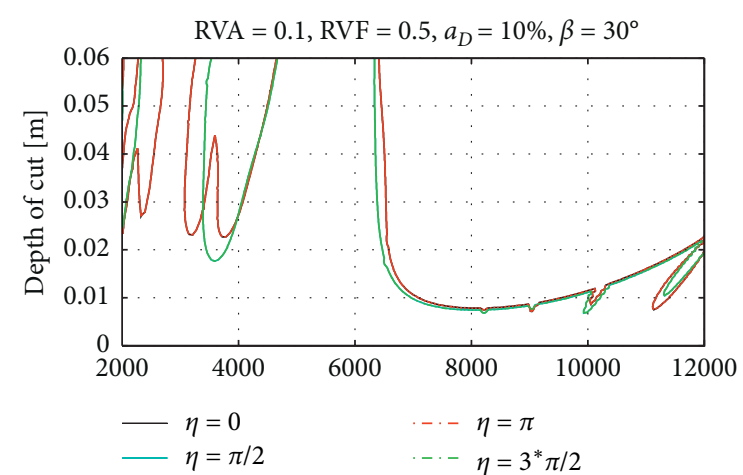

(b)

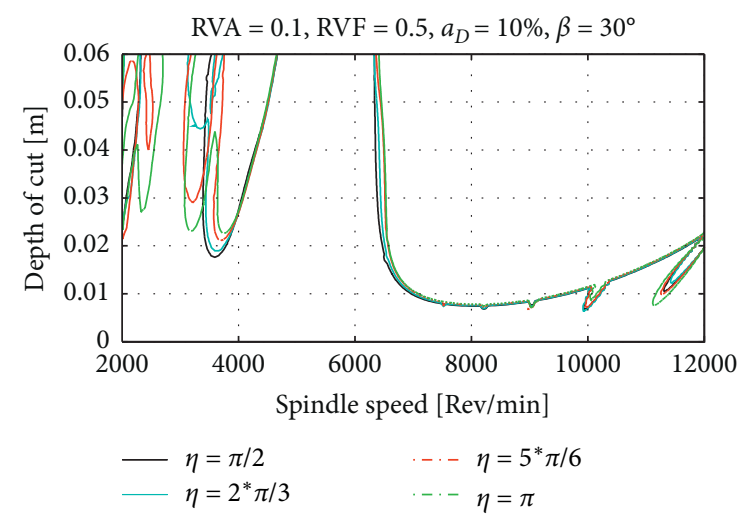

(d)

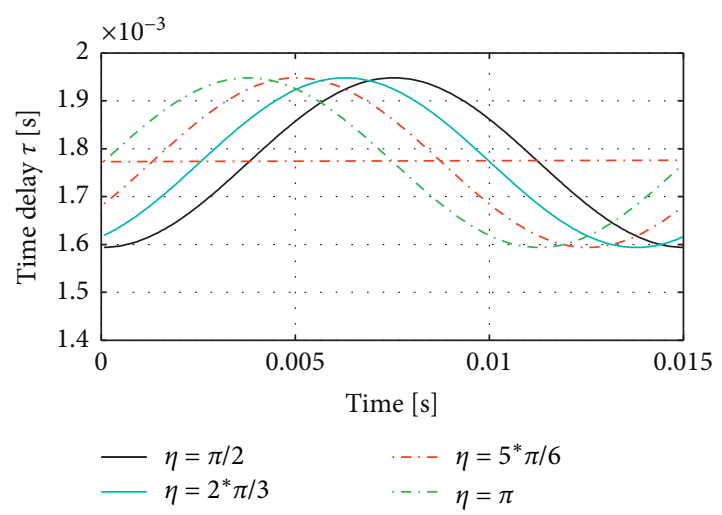

(f)

Figure 6: Influence of the process phase difference on cutting stability and time delays. (a-d) the SLDs under different process phase difference; $(e, f)$ the variation of the associated time delays.

take the median on the interval $\left[\eta_{a}, \eta_{b}\right]$ as the value of $\eta$ for obtaining a relatively compromised SLD; thus, the difference between the predicting SLDs and the actual ones can be minimized. With this in mind, $\eta$ can be defined as $\eta_{a}+\left(\eta_{a}-\eta_{b}\right) / 2$, that is, $\eta=3 n \pi / 4-\pi / 2$. Thus, some $\eta$, such as $\pi / 4$ and $5 \pi / 4$, are suggested and used in the practical application.

3.3. Influence of the Feed per Tooth. Feed per tooth is an important basis for the selection of cutting parameters [40, 41]. However, in most previous studies concerning milling chatter, it is ignored usually because it is considered to have no contribution to the dynamic cutting thickness and system stability. Also, one cannot get the effects of feed rate on the stability of milling by a linear cutting force model through the SMD in general. Here, the influence of feed per tooth on stability is investigated deeply, based on the method in this paper. Note that the following will be based on the nominal feed per tooth $f_{\mathrm{m}}$ in equation (10) instead of the time-varying one $f_{t, j}(t, \eta)$ for convenience.

To illustrate the performance of feed per tooth on the dynamic behaviour of the VPCVSS system, SLDs are here provided for four different feed per tooth (i.e., $f_{m}=0.01,0.05$, 0.1 , and $0.2 \mathrm{~mm} /$ tooth) under two different radial immersion ratios (10\% and 50\%), as shown in Figures 4(a) and 4(b). It 
can be seen that, with the increasing of $f_{m}$, the corresponding stability boundaries exhibit a trend of upward movement for both big and small radial depth of cut; thus, the limit depth of cut and stability area increases significantly. This is mainly due to the effect of the force-feed nonlinearity, specifically the nonlinear force coefficient $q$ in equation (2), on chatter in the milling process. Because the value of $q$ is equal to 0.75 , that is, a number less than 1 , a bigger $f_{m}$ will lead to a weaker nonlinear relationship between the cutting force and the dynamic displacements.

In order to further investigate the above effects, the SLDs corresponding to the four examples in Figure 4(a) are calculated under $q=0.94$ and shown in Figure 4(c). It can be seen from Figure 4(c) that the influence of feed per tooth is the same as that of $q=0.75$. However, compared with the latter, the former results in a more significant increase in stability. This is mainly due to the fact that since the values of $q$ are significantly less than unity, the nonlinear feed effect is more evident. The conclusions are consistent with those in [41], where the machining stability of the nonlinear feed is investigated for general turning and milling process through experiments and theoretical analysis. Thus, the combination nature in the VPCVSS system does not change the general influence mechanism of the feed per tooth on milling stability.

Here, it must be noted that the above analysis conclusions are based on the premise that the value of $q$ is less than one, which is suitable for some materials, such as aluminum alloy and steel [39]. However, if $q=1$, the force-feed relationship in equations (2) and (15) will become linear and the effect of the force-feed nonlinearity on the system dynamic mentioned above will vanish. At this time, only if some other conditions are added, the influence of the feed effect can be considered again. For example, when the multiple time delays caused by the tool runout is considered, a smaller feed will result in a higher stability boundary [15]. For the case of the variable time delay caused by the tool path, [41] exhibits a shift of SLDs with the change of feed. Further, if $q>1$, the associated conclusion in this article will be the opposite, that is, the increase of $f_{\mathrm{m}}$ results in the smaller limit depth of cut and stability area. Obviously, different considerations and analysis angles will lead to changes in conclusions; this is understandable.

Next, the simplification of the time-varying characteristic of the feed per tooth is carried out and its influence on the stability is investigated. For equation (10), if the VSS effect is not considered, that is, $\mathrm{RVA}=0$, it will degenerate to

$$
f_{t, j}(t, \eta)=\frac{f_{t m} N \psi_{j}}{360}
$$

if neither the VSS nor VPC effect is considered, it turns to

$$
f_{t, j}(t, \eta)=f_{t m}
$$

Figure 7 shows the SLDs corresponding to $f_{t, j}(t, \eta)$ respectively defined by equations (10), (29), and (30). It can be seen that there is a small difference among their stability boundaries for both the radial immersion ratios of $10 \%$ and $50 \%$. This indicates that no matter the multi-time-varying

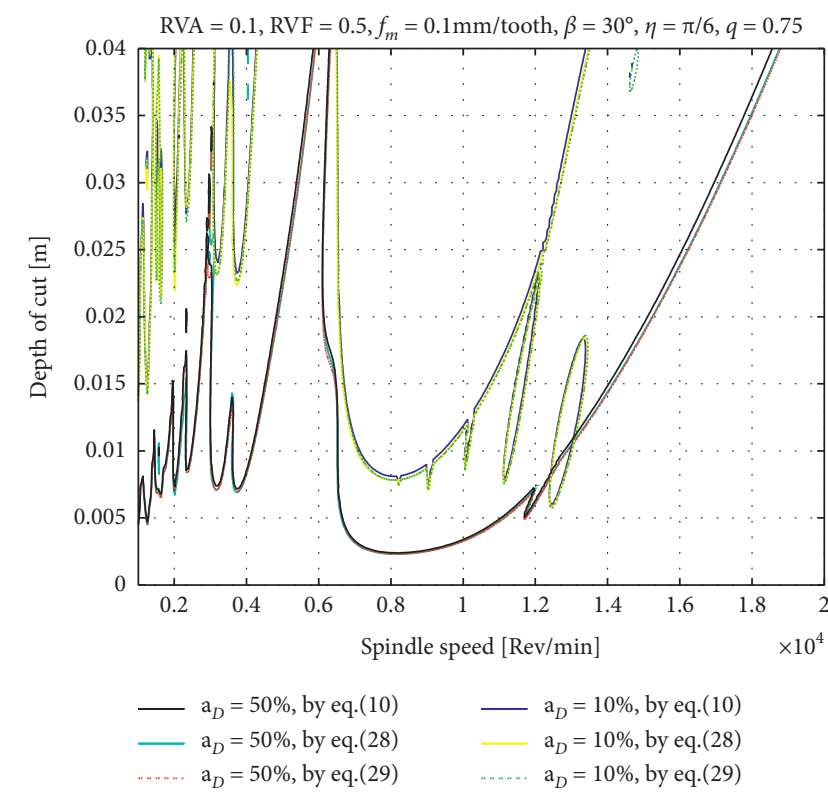

FIgURE 7: Effect of the different simplified forms of feed per tooth on milling stability.

characteristics of feed per tooth reflected by equation (10) or the multiconstant ones by equation (29), their influence on the stability is very limited. Thus, for the related general calculations, they both can be simplified to a single constant feed per tooth as shown in equation (30). However, this does not result in too much error for stability prediction.

3.4. Influence of the Helix Angle. Although many efforts have been devoted to studying the influence of helix angle on stability in the past, the related works are almost restricted to the regular cutter [33,34] or variable pitch cutter [19]; there is little work focusing on this topic for VSS milling, let alone VPCVSS. In this section, the above question will be analyzed in detail through combining some new calculations with the ones in Figure 3.

As shown in Figures $3(\mathrm{a})-3(\mathrm{~h})$, the stability limits increase evidently as the helix angle increases from $\beta=0^{\circ}$ to $30^{\circ}$. And this trend is more significant for the small axial depth of cut. This is attributed to the fact that larger helix angle means more obvious system time variation in small immersion ratios, which is characterized as highly interrupted. Further, it can be found that, for VSS milling the effect of helix angle is mainly reflected in the high-speed region (e.g., among 12000-18000 rpm in Figures 3(a)-3(d)), whereas this effect is relatively small for the low-speed region. However, the effect of helix angle is very significant in both high-speed and low-speed regions for VPCVSS milling, for example, $2000-6000 \mathrm{rpm}$ and $12000-18000 \mathrm{rpm}$ in Figures 3(e)-3(h). This may be attributed to the combined effect of VPC and VSS, that is, the positive effect of VPC (especially linear pitches case) on the maximum depth of cut in low speed as shown in [17] and the effective suppression of period double chatter in the high-speed region for VSS milling as shown in [28]. 


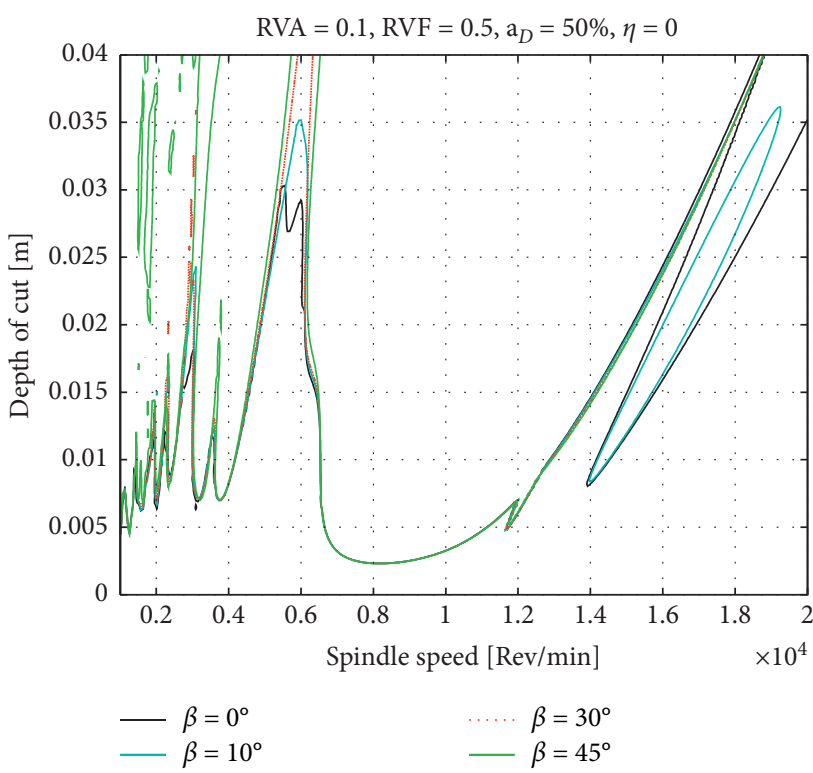

(a)

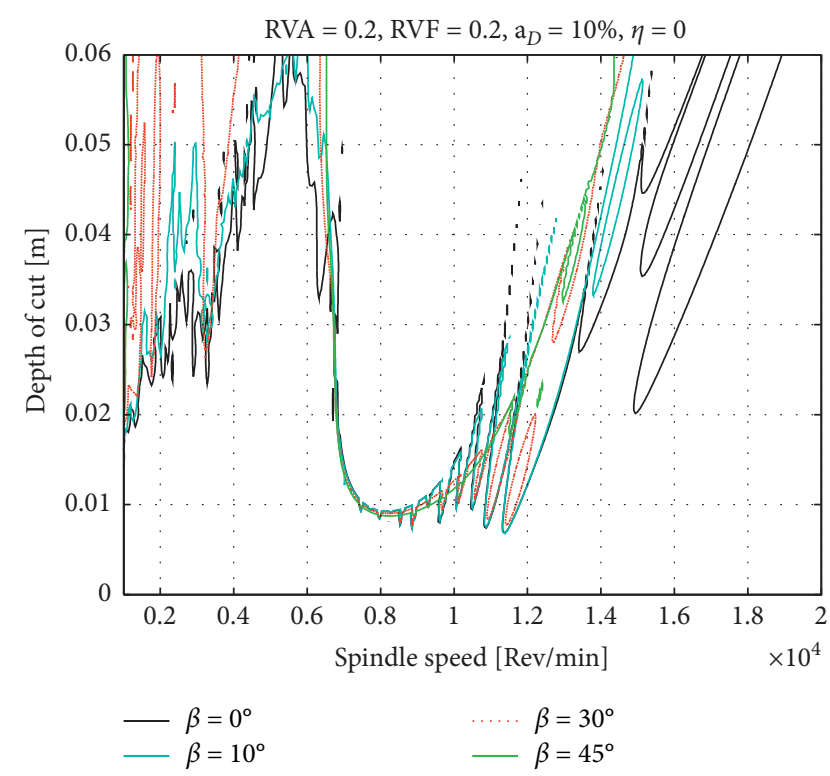

(b)

FIGURE 8: Effect of helix angle on cutting stability.

In order to evaluate the stability trend caused by helix angle in more detail, the calculations are conducted under four different helix angles (i.e., $\beta=0^{\circ}, 15^{\circ}, 30^{\circ}$, and $45^{\circ}$ ) and their results are shown in Figure 8. It can be seen that, with the increase of the helix angle, the stable cutting area increases significantly. In Figure 8(a), the so-called "stable islands" $[17,42]$ gradually appear around $2000 \mathrm{rpm}$ so that the original unstable region turns to stable, whereas the sharp appendages around $16000 \mathrm{rpm}$ gradually turn to "unstable islands" (flip bifurcation region) and disappear finally when $\beta=45^{\circ}$. However, it should be noted that, with the further increase of the helix angle, the influence of the helix angle on the cutting stability becomes weaker and weaker, especially in the high-speed area. This is mainly due to the smaller influence of the larger helix angle on the timevarying characteristics for the VPCVSS system.

\section{Conclusion}

This paper presents a dynamical model of the milling process of VPC and VSS considering the helix angle and the process phase difference. Then, an updated SDM is proposed to gain the stability chart. After the verification of the proposed method by comparisons with previously published works and the time-domain simulations, lots of influence analyses are conducted deeply. From the stability analysis, the following conclusions can be drawn:

(1) No matter for VSS or VPCVSS milling, the change of helix angle can result in significant stability difference for both small and big radial immersion ratios. However, for VSS milling, the effect of helix angle is mainly reflected in the high-speed region, whereas the effect of helix angle is very significant in both high-speed and low-speed regions for VPCVSS milling process.

(2) Process phase difference has an important influence on the cutting stability, especially for the low-speed domain. Under the influence of the phase difference, there are two kinds of change limits in SLDs, which occur in $\eta=(n-1) \pi$ and $n \pi / 2, n=1,2, \ldots$. Therefore, it is necessary to consider the phase difference in the stability calculation of VPCVSS or similar system. Considering the randomness and immeasurability of $\eta$ in the actual process, its recommended value is $\eta=3 n \pi / 4-\pi / 2$, such as $\pi / 4$ and $5 \pi / 4$.

(3) Due to the effect of the force-feed nonlinearity, as the feed per tooth is increasing, the stability boundaries exhibit a trend of upward movement, both for big and small radial immersion ratios, thus the limit depths of cut and stability area are increasing significantly.

(4) The multi-time-varying characteristic of feed per tooth in VPCVSS milling has almost negligible influence on the stability; thus, it can be simplified to a single constant one for a general calculation.

\section{Nomenclature}

A: $\quad$ State matrix for the complete system

$a_{p}: \quad$ Axial depth of cut $(\mathrm{m})$

$a_{D}: \quad$ Radial immersion ratio

$\mathbf{B}_{j}$ : $\quad$ State matrix for the system delays for the $j$-th tooth

$c_{x}$ : Damping coefficient

$F_{x}$ : $\quad$ Cutting force in $x$ direction 
$f_{m}: \quad$ Nominal feed per tooth ( $\mathrm{mm} /$ tooth)

$f_{t, j}$ : $\quad$ Feed per tooth of the system for the $j$-th tooth

$\mathrm{G}, \mathrm{g}$ : The new and traditional functions which determine whether the tooth is in contact with the workpiece

$h_{j}: \quad$ Instantaneous chip thickness (m)

$i$ : $\quad$ Index denoting flute (tooth) number

$j$ : $\quad$ Index denoting the number of the discrete time interval

$k$ : $\quad$ Number of discrete time intervals

$k_{r}, k_{t}$ : Tangential and radial directions cutting coefficients

$k_{x}: \quad$ Spring stiffness

$m_{x}: \quad$ Modal mass $(\mathrm{kg})$

$N$ : Number of teeth

Q: $\quad$ Nonlinear parameter in cutting force

$R: \quad$ Radius of a cutter $(\mathrm{m})$

RVA: Ratio of the speed variation amplitude to the nominal spindle speed

RVF: Ratio of the speed variation frequency to the nominal spindle speed

$T_{c}$ : $\quad$ Period of the VPCVSS system (s)

$T_{m}$ : $\quad$ Period of modulation by VSS (s)

$x, \dot{x}, \ddot{x}$ : Displacement, velocity, and acceleration in $x$ direction

$z: \quad$ Axial position on flute

$\beta$ : $\quad$ Helix angles $\left({ }^{\circ}\right)$

$\Delta t: \quad$ Time of discrete interval (s)

$\Delta \psi: \quad$ Variation of pitch angles $\left({ }^{\circ}\right)$

$\eta$ : $\quad$ Process phase difference between VPC and VSS (rad)

$\tau_{i, j}: \quad$ Approximated time delay in the $i$-th interval for $j$-th tooth (s)

$\phi_{e}, \phi_{s}: \quad$ Exit and start angles (rad)

$\phi_{\text {hex }}: \quad$ Exit angle difference caused by the helix angle (rad)

$\phi_{j}: \quad$ Angular position of $j$-th tooth

$\psi_{j}$ : $\quad$ Pitch angle between $j$-th and $(j-1)$-th teeth

$\Omega: \quad$ Spindle speed of VSS (Rev/min)

$\Omega_{0}: \quad$ Foundation speed of VSS (Rev/min)

$\Omega_{1}$ : Amplitude variation of spindle speed (Rev/min).

\section{Data Availability}

The MATLAB data used to support the findings of this study have been deposited in the "Baidu online disk" repository (https://pan.baidu.com/s/1_JvWniu1XP7TIbOCxjcrzw, extraction code: $x \operatorname{tgd})$.

\section{Conflicts of Interest}

The authors declare that there are no conflicts of interest regarding the publication of this paper.

\section{Acknowledgments}

This work was supported by the National Natural Science Foundation of China (Grant nos. 11702192 and 1405343), Tianjin Science and Technology Planning Project (Grant nos. 20JCYBJC00490, 18JCZDJC10050, 20JCQNJC01070, and 20JCQNJC01060).

\section{References}

[1] Y. Altintas and E. Budak, "Analytical prediction of stability lobes in milling," CIRP Annals. Manufacturing Technology, vol. 44, pp. 357-362, 1995.

[2] S. D. Merdol and Y. Altintas, "Multi frequency solution of chatter stability for low immersion milling," Journal of Manufacturing Science and Engineering, vol. 126, no. 3, pp. 459-466, 2004.

[3] P. V. Bayly, J. E. Halley, B. P. Mann, and M. A. Davies, "Stability of interrupted cutting by temporal finite element analysis," Journal of Manufacturing Science and Engineering, vol. 125, no. 2, pp. 220-225, 2003.

[4] E. A. Butcher and O. A. Bobrenkov, "On the Chebyshev spectral continuous time approximation for constant and periodic delay differential equations," Communications in Nonlinear Science and Numerical Simulation, vol. 16, no. 3, pp. 1541-1554, 2011.

[5] T. Insperger and G. Stepan, Semi-Discretization for TimeDelay Systems Stability and Engineering Applications, Springer-Verlag, New York, NY, USA, 1st edition, 2011.

[6] T. Insperger and G. Stépán, "Semi-discretization method for delayed systems," International Journal for Numerical Methods in Engineering, vol. 55, no. 5, pp. 503-518, 2002.

[7] T. Insperger and G. Stepan, "Updated semi-discretization method for periodic delay-differential equations with discrete delay," International Journal for Numerical Methods in Engineering, vol. 61, pp. 114-141, 2004.

[8] Y. Ding, L. Zhu, X. Zhang, and H. Ding, "A full-discretization method for prediction of milling stability," International Journal of Machine Tools and Manufacture, vol. 50, no. 5, pp. 502-509, 2010.

[9] Y. Liu, D. Zhang, and B. Wu, "An efficient full-discretization method for prediction of milling stability," International Journal of Machine Tools and Manufacture, vol. 63, pp. 40-48, 2012.

[10] D. Lehotzky, T. Inspergera, and G. Stepan, "Extension of the spectral element method for stability analysis of time-periodic delay-differential equations with multiple and distributed delays," Commun Nonlinear Sci, vol. 35, pp. 177-189, 2017.

[11] J. Slavicek, "The effect of irregular tooth pitch on stability of milling," in Proceedings of the 6th MTDR Conference, Pergamon Press, Manchester, UK, September 1965.

[12] Y. Altintas, S. Engin, and E. Budak, "Analytical stability prediction and design of variable pitch cutters," Journal of Manufacturing Science \& Engineering-ASME, vol. 121, pp. 173-179, 1999.

[13] E. Budak, "An analytical design method for milling cutters with nonconstant pitch to increase stability, Part I: theory," Journal of Manufacturing Science and Engineering, vol. 125, no. 1, pp. 29-34, 2003.

[14] N. Olgac and R. Sipahi, "Dynamics and stability of variablepitch milling," Journal of Vibration and Control, vol. 13, no. 7, pp. 1031-1043, 2007.

[15] M. Wan, W.-H. Zhang, J.-W. Dang, and Y. Yang, "A unified stability prediction method for milling process with multiple delays," International Journal of Machine Tools and Manufacture, vol. 50, no. 1, pp. 29-41, 2010.

[16] X. Zhang, C. Xiong, Y. Ding, and Y. Xiong, "Variable-step integration method for milling chatter stability prediction 
with multiple delays," Science China Technological Sciences, vol. 54, no. 12, pp. 3137-3154, 2011.

[17] G. Jin, H. Qi, Y. Cai, and Q. Zhang, "Stability prediction for milling process with multiple delays using an improved semidiscretization method," Mathematical Methods in the Applied Sciences, vol. 39, no. 4, pp. 949-958, 2016.

[18] G. Jin, Q. Zhang, S. Hao, and Q. Xie, "Stability prediction of milling process with variable pitch cutter," Mathematical Problems in Engineering, vol. 2013, no. 2, pp. 87-118, 2013.

[19] N. D. Sims, B. Mann, and S. Huyanan, "Analytical prediction of chatter stability for variable pitch and variable helix milling tools," Journal of Sound and Vibration, vol. 317, no. 3-5, pp. 664-686, 2008.

[20] N. Sims, "Fast chatter stability prediction for variable helix milling tools," Proceedings of The Institution of Mechanical Engineers Part C-Journal of Mechanical Engineering Science, vol. 230, no. 1, pp. 1989-1996, 2015.

[21] Z. Dombovari and G. Stepan, "The effect of helix angle variation on milling stability," Journal of Manufacturing Science \& Engineering-ASME, vol. 134, p. 51015, 2012.

[22] G. Jin, Q. Zhang, S. Hao, and Q. Xie, "Stability prediction of milling process with variable pitch and variable helix cutters," Proceedings of the Institution of Mechanical Engineers, Part C: Journal of Mechanical Engineering Science, vol. 228, no. 2, pp. 281-293, 2014.

[23] G. Jin, Q. Zhang, H. Qi, and B. Yan, "A frequency-domain solution for efficient stability prediction of variable helix cutters milling," Proceedings of the Institution of Mechanical Engineers, Part C: Journal of Mechanical Engineering Science, vol. 228, no. 15, pp. 2702-2710, 2014.

[24] A. R. Yusoff and N. D. Sims, "Optimisation of variable helix tool geometry for regenerative chatter mitigation," International Journal of Machine Tools and Manufacture, vol. 51, no. 2, pp. 133-141, 2011.

[25] Z. Dombovari, Y. Altintas, and G. Stepan, "The effect of serration on mechanics and stability of milling cutters," International Journal of Machine Tools and Manufacture, vol. 50, no. 6, pp. 511-520, 2010.

[26] T. Insperger and G. Stepan, "Stability analysis of turning with periodic spindle speed modulation via semidiscretization," Journal of Vibration and Control, vol. 10, no. 12, pp. 18351855, 2004.

[27] M. Zatarain, I. Bediaga, J. Muñoa, and R. Lizarralde, "Stability of milling processes with continuous spindle speed variation: analysis in the frequency and time domains, and experimental correlation," CIRP Annals, vol. 57, no. 1, pp. 379-384, 2008.

[28] S. Seguy, T. Insperger, L. Arnaud, G. Dessein, and G. Peign, "On the stability of high-speed milling with spindle speed variation," International Journal of Advanced Manufacturing Technology, vol. 48, no. 9-12, pp. 883-895, 2010.

[29] X. H. Long and B. Balachandran, "Stability of up-milling and down-milling operations with variable spindle speed," Journal of Vibration and Control, vol. 16, no. 7-8, pp. 1151-1168, 2011.

[30] Q. Xie and Q. Zhang, "Stability predictions of milling with variable spindle speed using an improved semi-discretization method," Mathematics and Computers in Simulation, vol. 85, no. 3, pp. 78-89, 2012.

[31] G. Totis, P. Albertelli, M. Sortino, and M. Monno, "Efficient evaluation of process stability in milling with spindle speed variation by using the Chebyshev collocation method," Journal of Sound and Vibration, vol. 333, no. 3, pp. 646-668, 2014.

[32] G. Jin, H. Qi, Z. Li, and J. Han, "Dynamic modeling and stability analysis for the combined milling system with variable pitch cutter and spindle speed variation," Communications in Nonlinear Science and Numerical Simulation, vol. 63, pp. 38-56, 2018.

[33] M. Zatarain, J. Muñoa, G. Peigné, and T. Insperger, "Analysis of the influence of mill helix angle on chatter stability," CIRP Annals, vol. 55, no. 1, pp. 365-368, 2006.

[34] B. Balachandran and M. X. Zhao, "A mechanics based model for study of dynamics of milling operations," Meccanica, vol. 35, no. 2, pp. 89-109, 2000.

[35] Y. Sun and S. Jiang, "Predictive modeling of chatter stability considering force-induced deformation effect in milling thinwalled parts," International Journal of Machine Tools and Manufacture, vol. 135, pp. 38-52, 2018.

[36] L. Zhang, B. Hao, D. Xu et al., "Dynamic milling stability prediction of thin-walled components based on VPC and VSS combined method," Journal of the Brazilian Society of Mechanical Sciences and Engineering, vol. 42, p. 6, 2020.

[37] S. Jiang and Y. Sun, "Stability analysis for a milling system considering multi-point-contact cross-axis mode coupling and cutter run-out effects," Mechanical Systems and Signal Processing, vol. 141, Article ID 106452, 2019.

[38] WA. Yang and C. Huang, "Stability analysis of 2-DOF milling dynamics for simultaneously varying tooth pitch and spindle speed with helix angle effect," International Journal of Advanced Manufacturing Technology, vol. 110, no. 5-6, pp. 1163-1177, 2020.

[39] K. B. Powell, "Cutting performance and stability of helical endmills with variable pitch," Dissertation, University of Florida, Gainesville, FL, USA, 2008.

[40] T. Insperger, D. A. W. Barton, and G. Stépán, "Criticality of hopf bifurcation in state-dependent delay model of turning processes," International Journal of Non-linear Mechanics, vol. 43, no. 2, pp. 140-149, 2008.

[41] R. G. Landers and A. G. Ulsoy, "Nonlinear feed effect in machining chatter analysis," Journal of Manufacturing Science \& Engineering-ASME, vol. 130, pp. 11017-11021, 2008.

[42] Q. Guo, Y. Jiang, B. Zhao et al., "Chatter modeling and stability lobes predicting for non-uniform helix tools," International Journal of Advanced Manufacturing Technology, vol. 87, no. 1-4, pp. 251-266, 2016. 